\title{
Range-free Sensor Positioning based on Bacterial Foraging Algorithm (BFO) in Wireless Sensor Networks
}

\author{
N. Pushpalatha \\ Assistant Professor, \\ Department of ECE, AITS, Tirupathi
}

\author{
B.Anuradha, $\mathrm{PhD}$ \\ Associate Professor, \\ Department of ECE, \\ S.V. University College of Engineering, Tirupathi
}

\begin{abstract}
In Wireless Sensor Network (WSN), the existing sensor positioning technique may result in increased cost, energy consumption, connectivity failure and less accuracy. In order to overcome these issues, in this paper, we propose a rangefree sensor positioning based on Bacterial Foraging Algorithm (BFO) in WSN. In this technique, initially the anchor nodes are placed using the coverage ratio. The coverage ratio depends on the network size. Then the anchor nodes use the BFO algorithm to estimate the distance between the unknown sensor nodes using neighbor density. BFO is a computational intelligence based technique that is not largely affected by the size and nonlinearity of the problem and can converge to the optimal solution in many problems where most analytical methods fail to converge. By simulation results, we show that the proposed technique enhances the accuracy and reduces the energy consumption.
\end{abstract}

\section{Keywords}

WSN, BFOA, Energy Consumption, Accuracy

\section{INTRODUCTION}

WIRELESS sensor networks (WSNs) are networks of distributed autonomous nodes that can sense or monitor physical or environmental conditions cooperatively [1]. Each sensor node consists of one or more sensors, a radio transceiver, a microcontroller and an energy source. Due to its potential applications in many areas ranging from environmental observation, natural habitat monitoring, medical, industry and military applications, WSN has attracted a lot of research interests in recent years [2].

In order to maximize the both capabilities of sensor networks, a novel deployment strategy should be provided [3]. The deployment of mobile sensor nodes in the region of interest (ROI) where interesting events might happen and the corresponding detection mechanism is required, is one of the key issues in this area. Before a sensor can provide useful data to the system, it must be deployed in a location that is contextually appropriate. Optimum placement of sensors results in the maximum possible utilization of the available sensors [4].

Sensor deployment strategies play a very important role in providing better QoS, which relates to the issue of how well each point in the sensing field is covered [5]. The most effective approach of sensor deployment is to place sensors in such a manner that the maximal network coverage is achieved [6]. An efficient deployment of sensor nodes will reduce the construction and communication cost of the network and improve the resource management [7] The deployment process is done according to only one or two constraints: i) deployment cost (number of sensors), ii) event detection probability, iii) connectivity, and iv) energy consumption (lifetime) [8]. Several deployment strategies have been studied for achieving an optimal sensor network architecture which would minimize cost, provides high sensing coverage, be resilient to random node failures, and so on.

Deployment of wireless sensor nodes can be categorized as static and the dynamic deployment. The static deployment involves choosing the best location according to the optimization strategy. It includes the deterministic deployment and the randomly deployment. In dynamic deployment, sensor nodes need automatically move to proper location before starting their work [14].

\section{PROBLEM IDENTIFICATION}

Efficient deployment strategies should achieve the following:

$$
\begin{aligned}
& >\text { Good connectivity } \\
& >\text { Maximal network coverage } \\
& >\text { Reduced construction and communication cost } \\
& >\text { Good event detection probability } \\
& >\text { Reduced energy consumption } \\
& >\text { Accurate positioning } \\
& >\text { Less position estimation error } \\
& >\text { Low complexity }
\end{aligned}
$$

According to the literature review in the previous section, the GSO based technique [7] considered only the distance between the sensor nodes. The ABC algorithm [10] considered only coverage rate. Though both the techniques provide accurate positioning, it lacks the other parameters. Though RSS-based positioning techniques in [11],[12] provide low complexity, they did not ensure energy and cost reduction. The sensor positioning scheme in [13] provides high accuracy and minimize the cost but if fails to achieve maximum coverage and connectivity.

Hence there is a need to develop sensor positioning technique which meet maximum of the above objectives.

In this paper, we propose to develop a range-free sensor positioning technique based on Bacterial Foraging Algorithm (BFO).

\section{RELATED WORKS}

Wen-Hwa Liao et al [7] have presented a sensor deployment design based on glowworm swarm optimization (GSO) which enhances the coverage after initial random deployment of the sensors. In this scheme, each sensor node is considered as individual glowworms emitting a luminant substance called luciferin and the intensity of the luciferin is reliant on the 
distance between the sensor node and its neighboring sensors. A sensor node is attracted towards its neighbors having lower intensity of luciferin and decides to move towards one of them. In this way, the coverage of the sensing field is maximized as the sensor nodes tend to move towards the region having lower sensor density.

While GSO has been applied by Wen-Hwa Liao et al [7], Celal Ozturk et al [10] have used the artificial bee colony (ABC) algorithm for the dynamic deployment of stationary and mobile sensor networks. A probabilistic detection model is considered to obtain more realistic results while computing the effectively covered area. The aim of the optimization technique is to maximize the coverage rate of the network by estimating the coverage ratio.

Naveed Salman et al [11] have improved the performance of the Received Signal Strength (RSS)-based localization technique. It proposed the weighted least squares (WLS) algorithm to improve the location estimation accuracy. To further improve the performance of WLS algorithm, a reference anchor optimization using minimization of the theoretical mean square error (MSE) is also proposed.

A Three-dimensional accurate positioning algorithm has been proposed by Quan Liu et al [12]. It uses the RSSI-based positioning method which constructs an attenuation model of wireless signal between beacon node and unknown node in three-dimensional space. It compensates for the loss of wireless signal on the basis of their material, thickness and other factors when there are obstacles or walls. The error revision algorithm is used to improve the positioning accuracy.

Junho Park et al [13] have presented a novel sensor positioning scheme in non-uniform wireless sensor networks to ensure high accuracy of sensor positioning. In this scheme, minimum anchor nodes are used and the distance is estimated according to the neighbor density in the non-uniform sensor network environments. It uses at least 4 anchor nodes placed at the boundary of the sensing fields to minimize the cost of construction of the sensor network.

\section{PROPOSED SOLUTION}

\subsection{Overview}

In this paper, we propose a range-free sensor positioning based on Bacterial Foraging Algorithm (BFO) in WSN. In this technique, initially the anchor nodes are placed using the coverage ratio. The coverage ratio depends on the network size. Then the anchor nodes use the BFO algorithm to estimate the distance between the unknown sensor nodes using neighbor density.

\subsection{Estimation of Coverage Ratio}

Each sensor is aware of its position. It communicates with other sensor nodes and the mobile nodes can change their positions by using the other nodes information.

The coverage ratio $(\beta)$ of the sensor network is estimated using the following equation (1)

$$
\beta=\frac{C S_{i}}{Z} \text {, i } \varepsilon \mathrm{X}
$$

where $\mathrm{CS}_{\mathrm{i}}=$ coverage of a sensor $\mathrm{i}$
$X=$ set of the nodes

$\mathrm{Z}=$ total size (It is based on the network space considered)

\subsection{Basics of Bacteria Foraging Optimization Algorithm}

The Bacterial Foraging algorithm is a computational intelligence based technique that is not largely affected by the size and nonlinearity of the problem and can converge to the optimal solution in many problems where most analytical methods fail to converge [15]. It has been widely accepted as a global optimization algorithm of current interest for distributed optimization and control which is inspired by the social foraging behavior of Escherichia coli.

For a real bacterium, during foraging, the locomotion (tumble or swim) is attained through a set of tensile flagella. The main function of flagella is to assist E.Coli bacterium during its locomotion. When they rotate the flagella in the clockwise direction, each flagellum pulls on the cell. This causes the flagella to move independently and at the end the bacterium tumbles with minimum tumbling count. However in a harmful place, it tumbles frequently to search nutrient gradient. The process of moving the flagella in the counter clockwise direction helps the bacterium to swim at very fast rate.

In this algorithm, the bacterium endures chemotaxis, where they tend to move towards a nutrient gradient and prevents noxious environment. In general, the bacterium moves for a longer distance in a friendly environment. When they receive sufficient food, they increase in length and in presence of suitable temperature they break in the middle to form its exact replica.

If there is a sudden environmental change or attack, the chemotactic process may get destroyed and there is a possibility for the group of bacteria to move to other location. This refers to the elimination-dispersal in the real bacterial population. During this phase, all the bacteria in a region are killed or a group is dispersed into a new part of the environment.

4.3 Range-Free Sensor Positioning Based on Bacterial Foraging Algorithm (BFO)

Initially the anchor or beacon nodes are placed using the coverage ratio $(\beta)$. The anchor nodes then use the BFO algorithm to estimate the distance between the unknown sensor nodes using the neighbor density.

Let $\mathrm{B}$ be the number of anchor nodes

Let $X$ be the number of steps

Let $\mathrm{Y}_{\mathrm{y}}$ and $\mathrm{P}_{\mathrm{y}}$ be the number of chemotactic steps and its probability respectively,

Let $\mathrm{Z}_{\mathrm{z}}$ and $\mathrm{P}_{\mathrm{z}}$ be the reproduction steps and its probability respectively

Let $\mathrm{E}_{\mathrm{e}}$ and $\mathrm{P}_{\mathrm{e}}$ be the number of elimination disperses steps and its probability respectively.

Let $\mathrm{S}$ (i) be the step size, where $\mathrm{i}=1,2, \ldots, \mathrm{B}$

Let $\alpha^{i}$ be the position of the anchors, $\mathrm{i}=1,2, \ldots \ldots, \mathrm{B}$

Let $\mathrm{F} \alpha$ be the combined factor of attractants and repellants of the nodes from the network, where $\mathrm{F}$ represents the gradient.

i.e. If $\mathrm{F} \alpha<0$, then the anchor nodes are in active environment.

F $\alpha=0$, then the anchor nodes are in neutral environment. 

environment.

F $\alpha>0$, then the anchor nodes are in harmful

Let $\mathrm{M} \mathrm{y}, \mathrm{z}, \mathrm{e}=\alpha^{i} \mathrm{y}, \mathrm{z}, \mathrm{e}, \mathrm{i}=1,2, \ldots . \mathrm{B}$

The above equation reveals the position of each member in the population of $\mathrm{S}$ anchor nodes, at respective event

Let $\mathrm{LT}_{\mathrm{B}}$ be the lifetime of the anchor nodes estimated during chemotactic steps.

Let $\mathrm{Y} \varpi>0$ represent the basic chemotactic step size which is used to define the length of steps during runs.

Let $\varpi \alpha$ be the unit length random direction representing tumble. (Using unit length, the direction of movement after a tumble can be estimated.)

The steps involved in this algorithm are as follows:

1. Compute $\alpha$ which is the control and is randomly distributed across the nodes.

2. Elimination - Dispersal Loop: $\mathrm{e}=\mathrm{e}+1$

3. Reproduction loop $=\mathrm{z}=\mathrm{z}+1$

4. Chemotaxis loop: $y=y+1$

- Chemotaxis simulates the movement of anchor nodes which is estimated as follows: For $\mathrm{i}=1,2, \ldots \mathrm{B}$

- $\quad$ Estimate the objective function $\mathrm{Y}(\mathrm{i}, \mathrm{y}, \mathrm{z}, \mathrm{e})$

Let $\mathrm{Y}(\mathrm{i}, \mathrm{y}, \mathrm{z}, \mathrm{e})=\mathrm{Y}(\mathrm{i}, \mathrm{y}, \mathrm{z}, \mathrm{e})+\mathrm{Y}_{\mathrm{ss}}\left(\alpha^{i}(\mathrm{y}, \mathrm{z}, \mathrm{e}), \mathrm{P}(\mathrm{y}, \mathrm{z}, \mathrm{e})\right)$

Where $\mathrm{Y}_{\mathrm{ss}}\left(\alpha^{i}(\mathrm{y}, \mathrm{z}, \mathrm{e})\right)=$ objective function value represented as attractant and repellent to be added to the actual objective function

Let $\mathrm{Y}_{\text {last }}=\mathrm{Y}(\mathrm{i}, \mathrm{y}, \mathrm{z}, \mathrm{e})$ to save this value as the better solution need to be found through execution

- Tumble: Generate a random vector $\Delta \varpi \varepsilon \mathrm{Q}^{\mathrm{p}}$, With each element $\Delta_{\mathrm{v}} \varpi, \mathrm{p}=1,2, \ldots \mathrm{n}$

- $\quad$ Move let $\alpha^{i}(\mathrm{y}+1, \mathrm{z}, \mathrm{e})=\alpha^{i}(\mathrm{y}, \mathrm{z}, \mathrm{e})+\mathrm{S}$ (i) $\frac{\Delta(i)}{\sqrt{\Delta^{t}(i) i \Delta(i)}}$

This reveals the step size S (i) in the direction of tumble for anchor nodes $\mathrm{i}$.

- Estimate next objective function $\mathrm{Y}(\mathrm{i}, \mathrm{y}+1, \mathrm{z}, \mathrm{e})$

$\mathrm{Y}(\mathrm{i}, \mathrm{y}+1, \mathrm{z}, \mathrm{e})=\mathrm{Y}(\mathrm{i}, \mathrm{y}, \mathrm{z}, \mathrm{e})+\mathrm{Y}_{\mathrm{ss}}\left(\alpha^{i}(\mathrm{y}+1, \mathrm{z}, \mathrm{e}), \mathrm{P}(\mathrm{y}+1\right.$, $\mathrm{z}, \mathrm{e}))$

- Estimate the neighbor nodes density. If the density is minimum, then Go to next step else Go to reproduction step

- $\quad$ Swim

Let $\mathrm{v}=0$ (counter for swim length)

While $\mathrm{v}<\mathrm{X}$

Let $\mathrm{v}=\mathrm{v}+1$

If $\mathrm{Y}(\mathrm{i}, \mathrm{y}+1, \mathrm{z}, \mathrm{e})=\mathrm{Y}_{\text {last }}$ and
Let $\alpha^{i}(\mathrm{y}+1, \mathrm{z}, \mathrm{e})=\alpha^{i}(\mathrm{y}, \mathrm{z}, \mathrm{e})+\mathrm{S}(\mathrm{i}) \frac{\Delta(i)}{\sqrt{\Delta^{t}(i) i \Delta(i)}}$ and

use $\alpha^{i}(\mathrm{y}+1, \mathrm{z}, \mathrm{e})$ to estimate $\mathrm{Y}(\mathrm{i}, \mathrm{y}+1, \mathrm{z}, \mathrm{e})$

Else

Let $\mathrm{v}=\mathrm{X}$, end of while statement

Go to next anchor node $\mathrm{i}+1$ if $\mathrm{i} \neq \mathrm{S}$

- If $\mathrm{y}<\mathrm{Y}$, go to reproduction stage. Otherwise continue chemotaxis as the node is still active.

5. Reproduction

For a given $\mathrm{z}$ and $\mathrm{e}$, and for each $\mathrm{i}=1,2, \ldots \mathrm{B}$

Let $Y_{A C T I V E}^{i}=\sum_{y=1}^{Y+1} Y \varpi, y, z, e$ be the strength of the node i.

- $\quad$ Sort the $X$ anchor nodes and chemotactic parameter $\mathrm{S}$ (i) in order of ascending value of $Y_{\text {ACTIVE }}^{i}$

- The nodes with high $Y_{\text {ACTIVE }}^{i}$ value dies and other nodes with best values split into two.

- If $\mathrm{z}<\mathrm{Z}$, go to elimination step. In this case, we have not reached the number of specified reproduction steps

6. Elimination Dispersal

For $\mathrm{i}=1,2, \ldots, \mathrm{X}$ with probability $\mathrm{P}_{\mathrm{e}}$, eliminate and disperse each anchor nodes, eliminate node and disperse one to a random location on the optimization domain.

- If $\mathrm{e}<\mathrm{P}_{\mathrm{e}}$, then go to step 1. Otherwise terminate the event.

Thus, based on the neighbor node density, the anchor nodes estimate the distance between the unknown sensor nodes.

Figure 1 demonstrates the anchor node deployment in sensor network

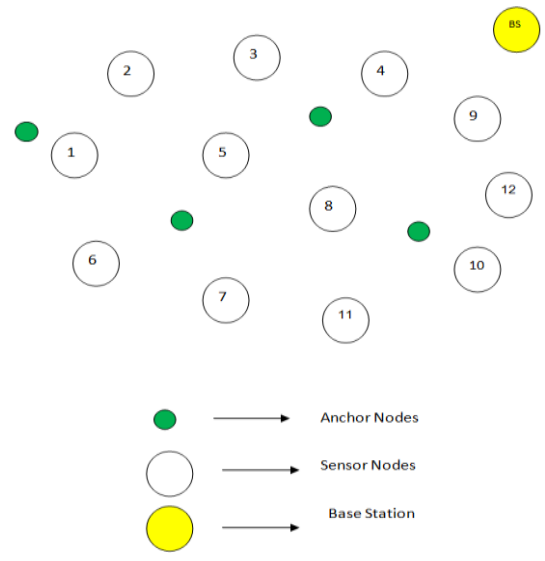

Fig1AnchorNode Deployment 


\section{SIMULATION RESULTS}

\subsection{Simulation Parameters}

We use NS2 [17] to simulate our proposed Range-free Sensor Positioning Based on Bacterial Foraging Algorithm (RSPBFA) protocol. We use the IEEE 802.11 for wireless Sensor Networks as the MAC layer protocol. It has the functionality to notify the network layer about link breakage. In our simulation, the number of nodes is varied as $50,100,150$ and 200 . The area size is 500 meter $x 500$ meter square region for 50 seconds simulation time. The simulated traffic is Constant Bit Rate (CBR).

Our simulation settings and parameters are summarized in table 1

Table 1: Simulation parameters

\begin{tabular}{|l|l|}
\hline No. of Nodes & $50,100,150$ and 200 \\
\hline Area & 500 X 500 \\
\hline MAC & 802.11 \\
\hline Simulation Time & $50 \mathrm{sec}$ \\
\hline Traffic Source & CBR \\
\hline Rate & $50 \mathrm{~Kb}$ \\
\hline Propagation & TwoRayGround \\
\hline Antenna & OmniAntenna \\
\hline Range & $250,300,350$ and $400 \mathrm{~m} / \mathrm{s}$ \\
\hline Initial Energy & $10.3 \mathrm{~J}$ \\
\hline Transmission Power & 0.660 \\
\hline Receiving Power & 0.395 \\
\hline
\end{tabular}

\subsection{Performance Metrics}

We evaluate performance of the new protocol mainly according to the following parameters. We compare the ABC [10] algorithm with our proposed RSPBFA protocol.

Average Packet Delivery Ratio: It is the ratio of the number of packets received successfully and the total number of packets transmitted.

Average end-to-end delay: The end-to-end-delay is averaged over all surviving data packets from the sources to the destinations.

Residual Energy: It is the amount of energy remain in the nodes after the data transmission

Packet Drop: It is the number of packets dropped during the data transmission

\subsection{Results \& Analysis}

The simulation results are presented in the next section.

\subsubsection{Based on Nodes}

In our first experiment we are number of nodes as $50,100,150$ and 200.

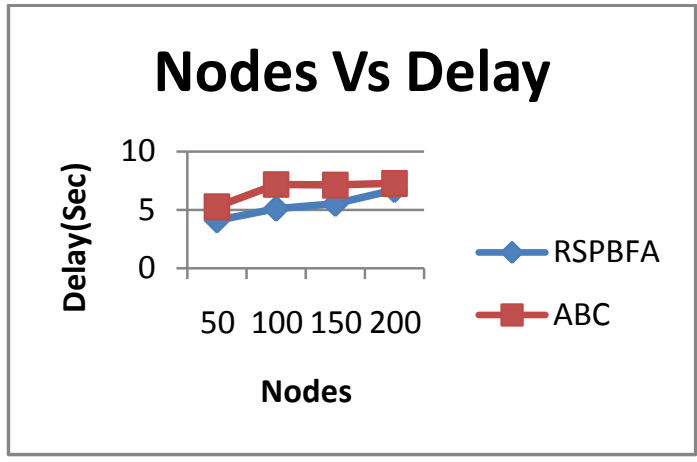

Fig 2: Nodes Vs Delay

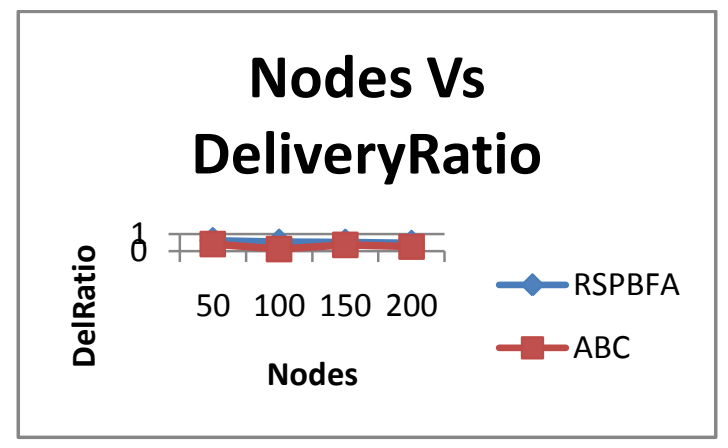

Fig 3: Nodes Vs Delivery Ratio

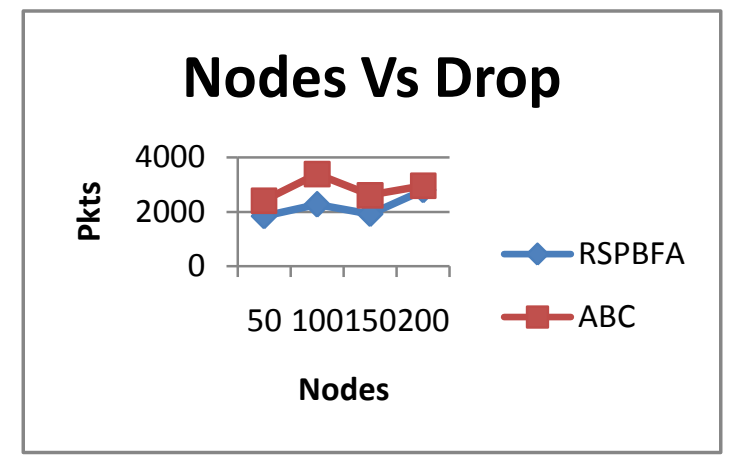

Fig 4: Nodes Vs Drop

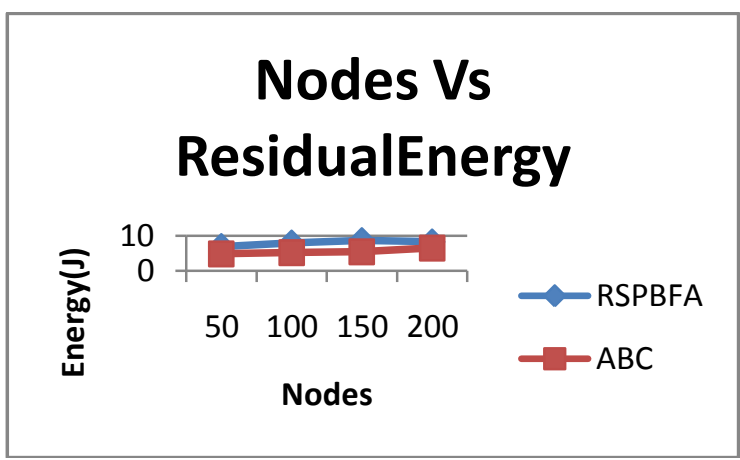

Fig 5: Nodes Vs Residual Energy

Figures 2 to 5 show the results of delay, delivery ratio, packet drop and Residual energy by varying the number of nodes as $50,100,150$ and 200 for the CBR traffic in RSPBFA and ABC protocols. When comparing the performance of the two protocols, we infer that RSPBFA outperforms ABC by $21 \%$ in terms of delay, $48 \%$ in terms of delivery ratio, $22 \%$ in terms of packet drop and $31 \%$ in terms of energy consumption. 


\subsubsection{Based on Range}

In our second experiment we vary the transmission range as $250,300,350$ and $400 \mathrm{~m} / \mathrm{s}$.

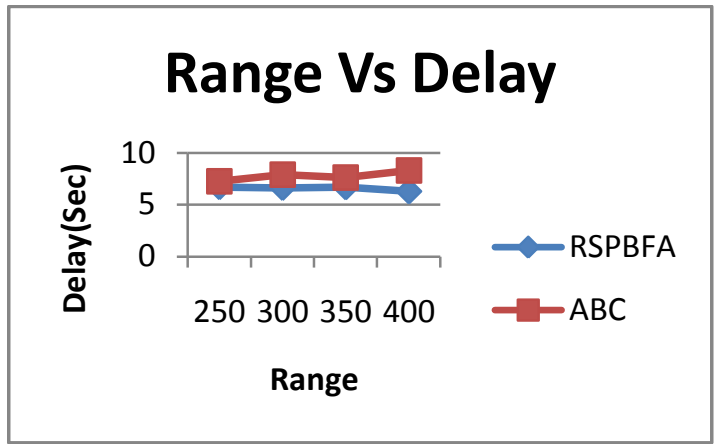

Fig 6: Range Vs Delay

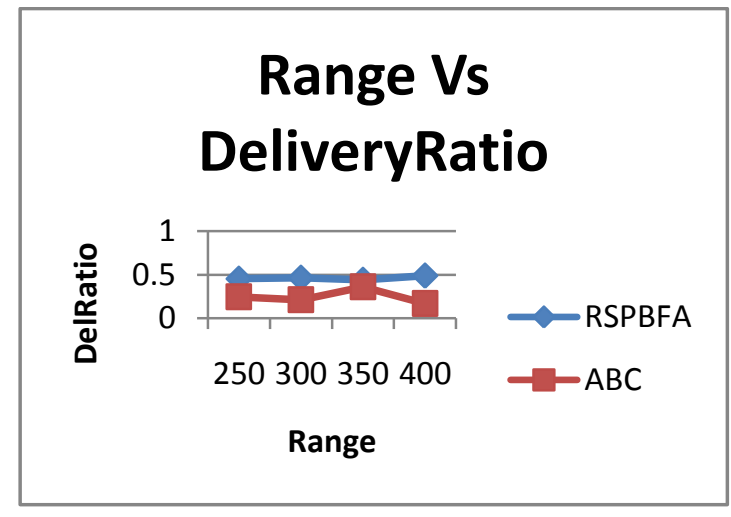

Fig 7: Range Vs Delivery Ratio

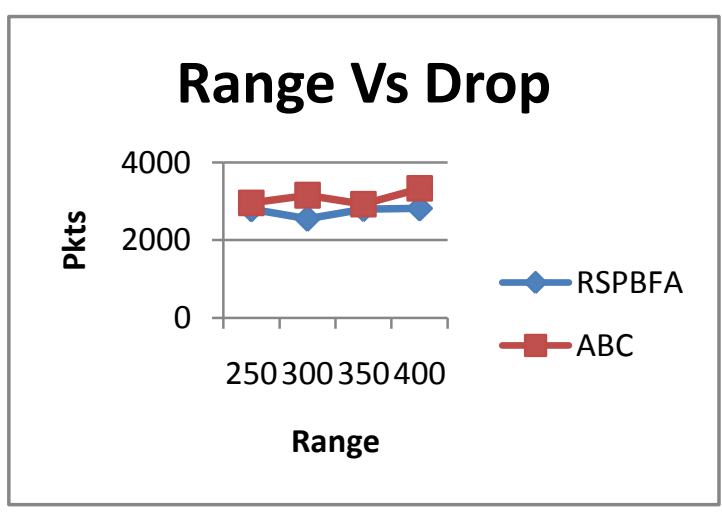

Fig 8: Range Vs Drop

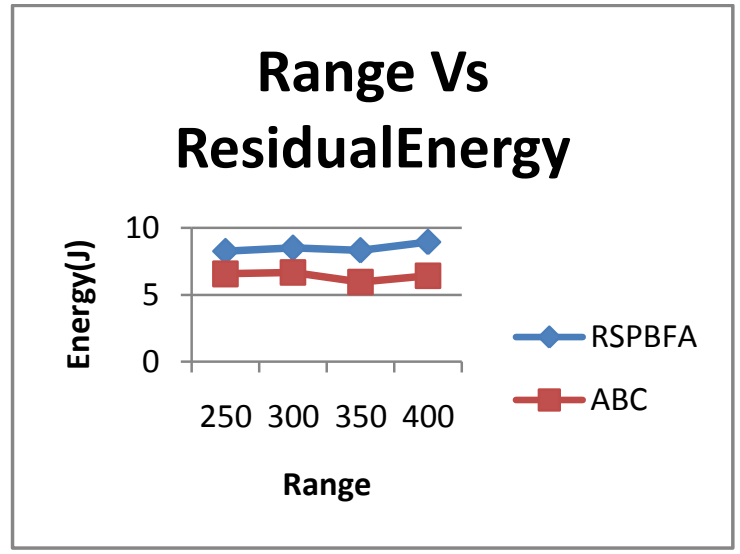

Fig 9: Range VS Residual Energy

Figures 6 to 9 show the results of delay, delivery ratio, packet drop and Residual energy by varying the range as 250,300,350 and 400 for the CBR traffic in RSPBFA and $\mathrm{ABC}$ protocols. When comparing the performance of the two protocols, we infer that RSPBFA outperforms $\mathrm{ABC}$ by $15 \%$ in terms of delay, $46 \%$ in terms of delivery ratio, $11 \%$ in terms of packet drop and $25 \%$ in terms of energy consumption.

\section{CONCLUSION}

In this paper, we have proposed a range-free sensor positioning based on Bacterial Foraging Algorithm (BFO) in WSN. In this technique, initially the anchor nodes are placed using the coverage ratio. The coverage ratio depends on the network size. Then the anchor nodes use the BFO algorithm to estimate the distance between the unknown sensor nodes using neighbor density. BFO is a computational intelligence based technique that is not largely affected by the size and nonlinearity of the problem and can converge to the optimal solution in many problems where most analytical methods fail to converge. By simulation results, we have shown that the proposed technique enhances the accuracy and reduces the energy consumption.

\section{REFERENCES}

[1] Raghavendra V. Kulkarni, and Ganesh Kumar Venayagamoorthy, "Bio-inspired Algorithms for Autonomous Deployment and Localization of Sensor Nodes", IEEE Transactions on Systems, Man, and Cybernetics-Part C: Applications and Reviews, Vol. 40, No. 6, NOV. 2010

[2] HA Nguyen, K S Low, H Guo, "Real Time Determination of Sensor Node Location in a Wireless Sensor Network using Particle Swarm Optimization", Proceedings of the 10th WSEAS International Conference on EVOLUTIONARY COMPUTING,pp.140-145,2009.

[3] Hyungmin Park, Ji-Hyeong Han and Jong-Hwan Kim, "Swarm Intelligence-based Sensor Network Deployment Strategy", IEEE World Congress on Computational Intelligence,pp.4210-4215, July, 2010.

[4] Wu Xiaoling, Shu Lei, Wang Jin, Jinsung Cho1, and Sungyoung Lee, "Energy-efficient Deployment of Mobile Sensor Networks by PSO", AP Web Workshops, 2006. 
[5] Amjad Osmani, "Design and evaluation of two distributed methods for sensors placement in Wireless Sensor Networks", Journal of Advances in Computer Research, pp.13-26, 2011

[6] Nikitha Kukunuru, Babu Rao Thella, Rajya Lakshmi Davuluri, "Sensor Deployment Using Particle Swarm Optimization", International Journal of Engineering Science and Technology, Vol. 2(10), pp. 5395-5401, 2010.

[7] Wen-Hwa Liao, Yucheng Kao, Ying-Shan Li, “A sensor deployment approach using glowworm swarm optimization algorithm in wireless sensor networks", Expert Systems with Applications,pp.12180-12188,2011.

[8] Nadjib Aitsaadi, Nadjib Achir, Khaled Boussetta and Guy Pujolle, "Multi-Objective WSN Deployment: Quality of Monitoring, Connectivity and Lifetime",IEEE ICC proceedings, 2010.

[9] Roghayeh Soleimanzadeh, Bahareh J. Farahani and Mahmood Fathy," PSO based Deployment Algorithms in Hybrid Sensor Networks", IJCSNS International Journal of Computer Science and Network Security, VOL.10 No.7, July 2010

[10] Celal Ozturk , Dervis Karaboga and Beyza Gorkemi," Probabilistic Dynamic Deployment of Wireless Sensor Networks by Artificial Bee Colony Algorithm", Sensors 2011, 11, 6056-6065

[11] Naveed Salman, Mounir Ghogho and A. H. Kemp,"Optimized Low Complexity Sensor Node Positioning in Wireless Sensor Networks", IEEE 2013.

[12] Quan LIU, Ping REN and Zude ZHOU," Threedimensional Accurate Positioning Algorithm based on Wireless Sensor Networks", JOURNAL OF COMPUTERS, VOL. 6, NO. 12, DECEMBER 2011

[13] Junho Park, Hyuk Park, Dong-ook Seong and Jaesoo Yoo,"A Sensor Positioning Scheme with High Accuracy in Nonuniform Wireless Sensor Networks", International Journal of Distributed Sensor Networks,Volume 2013, Article ID 507605, 7 pages

[14] Haitao Zhang and Cuiping Liu,"A Review on Node Deployment of Wireless Sensor Network", IJCSI International Journal of Computer Science Issues, Vol. 9, Issue 6, No 3, November 2012

[15] M.Senthil Kumar and Dr.P.Renuga, "Bacterial Foraging Algorithm based Enhancement of Voltage Profile and Minimization of Losses Using Thyristor Controlled Series Capacitor (TCSC)", International Journal of Computer Applications (0975 - 8887), Volume 7- No.2, September 2010

[16] Swagatam Das, Arijit Biswas, Sambarta Dasgupta and Ajith Abraham,"Bacterial Foraging Optimization Algorithm: Theoretical Foundations, Analysis, and Applications

[17] Network Simulator: http:///www.isi.edu/nsnam/ns

\section{AUTHOR PROFILE}

N.Pushpalatha completed her B.Tech at JNTU, Hyderabad in 2004 and M.Tech at A.I.T.S., Rajampet in 2007. Presently she is working as Assistant Professor of ECE, Annamacharya Institute of Technology and Sciences Tirupati since 2006. She has guided many B.Tech projects and M.Tech Projects. Her Research area includes Data Communications and Ad-hoc Wireless Sensor Networks.

Dr.B.Anuradha is working as Professor in the Department of ECE, at Sri Venkateswara University College of Engineering since 1992. She has guided many B.Tech and M.Tech projects. At present Five Scholars are working for $\mathrm{PhD}$. She has published a good number of papers in journals and conferences. 